\title{
First clinical experience of the looped Inoue balloon technique for antegrade percutaneous balloon aortic valvuloplasty.
}

\section{$\operatorname{AUTHOR}(\mathrm{S}):$}

Yano, Mariko; Saito, Naritatsu; Watanabe, Shin; Watanabe, Hirotoshi; Nishikawa, Ryusuke; Fujino, Takeshi; Bao, Bingyuan; ... Sakata, Yoshihito; Kimura, Takeshi; Inoue, Kanji

\section{CITATION:}

Yano, Mariko ...[et al]. First clinical experience of the looped Inoue balloon technique for antegrade percutaneous balloon aortic valvuloplasty.. Heart and vessels 2015, 30(6): 830834

\section{ISSUE DATE:}

2015-11

URL:

http://hdl.handle.net/2433/207524

\section{RIGHT:}

The final publication is available at Springer via http://dx.doi.org/10.1007/s00380-014-0552-1.: The full-text file will be made open to the public on 1 November 2016 in accordance with publisher's 'Terms and Conditions for Self-Archiving'.; この論文は出版社版でありません。引用の際には出版社版をご確認じ利用ください。; This is not the published version. Please cite only the published version. 


\section{First Clinical Experience of the Looped Inoue Balloon Technique for Antegrade Percutaneous Balloon Aortic Valvuloplasty}

Mariko Yano, M.D. ${ }^{1}$, Naritatsu Saito, M.D. ${ }^{1}$, Shin Watanabe M.D. ${ }^{1}$, Hirotoshi Watanabe M.D. ${ }^{1}$, Ryusuke Nishikawa, M.D. ${ }^{1}$, Takeshi Fujino, M.D. ${ }^{1}$, Bingyuan Bao, M.D. ${ }^{1}$, Erika Yamamoto, M.D. ${ }^{1}$, Hiroki Watanabe, M.D. ${ }^{1}$, Kenji Nakatsuma, M.D.1, Masao Imai, M.D. ${ }^{1}$, Takeru Makiyama, M.D. ${ }^{1}$, Yoshihito Sakata, M.D. ${ }^{2}$, Takeshi Kimura, M.D. ${ }^{1}$, Kanji Inoue, M.D. ${ }^{3}$

1. Department of Cardiovascular Medicine, Graduate School of Medicine, Kyoto University, Kyoto, Japan

2. Heart Center, Ikegami General Hospital, Tokyo, Japan

3. PTMC Institute, Kyoto, Japan

\section{Address for Correspondence:}

Naritatsu Saito

Department of Cardiovascular Medicine, Graduate School of Medicine, Kyoto University

54 Shogoin Kawahara-cho, Sakyo-ku, Kyoto 606-8507, Japan 
E-mail: naritatu@kuhp.kyoto-u.ac.jp

Tel: +81-75-751-3198, Fax: +81-75-751-3299

\section{Abstract: 179 words}

Balloon aortic valvuloplasty (BAV) has played a limited role in the management of patients with severe aortic stenosis. However, BAV is being performed more frequently these days with the emergence of transcatheter aortic valve implantation (TAVI). We previously described a technique named "looped Inoue balloon technique" to simplify the antegrade transvenous BAV by making a loop in the left atrium using two stylets. We present a case in which the looped Inoue balloon technique was successfully applied. The patient was an 83-year-old woman with progressive dyspnea due to severe aortic stenosis. The aortic valve area was $0.39 \mathrm{~cm}^{2}$ with a mean transvalvular gradient of $46 \mathrm{mmHg}$. The patient was deemed high risk for surgical aortic valve replacement or TAVI in view of the multiple comorbidities and frailty. Antegrade BAV using the looped Inoue balloon technique was performed. The procedure was successful without any complications. The post procedural aortic valve area increased to $1.15 \mathrm{~cm}^{2}$ with a mean pressure gradient of 23 $\mathrm{mmHg}$. This is the first report of clinical use of the looped Inoue balloon technique for antegrade BAV, 
Key Words: Balloon Aortic Valvuloplasty, Aortic Valve Stenosis, Structural Heart Disease

\section{Main Texts: 1762 words}

\section{Introduction:}

Balloon aortic valvuloplasty (BAV) has played a niche role in the treatment of patients with severe aortic stenosis. After the introduction of transcatheter aortic valve implantation (TAVI), BAV is more frequently used as a bridge therapy to TAVI[1, 2]. BAV can be performed by either a retrograde transarterial approach or an antegrade transvenous approach. The antegrade transvenous approach has several advantages including reduced vascular complication, reduction of stroke, and greater increase of post procedural valve area[3]. However, the antegrade approach is more technically complicated and demanding[3-5]. We previously described a novel technique to simplify the antegrade BAV, which utilizes an originally customized Inoue balloon[6]. The customized Inoue balloon catheter is designed to make a loop in the left atrium, then advanced into the left ventricle while keeping the loop. The technique is named as the looped Inoue balloon technique, and 
its feasibility has been proven in pre-clinical animal experiment[6]. In the present paper, we describe the first clinical use of the looped Inoue balloon technique.

\section{Case Report:}

An 83-year-old female with progressive dyspnea in New York Heart Association functional class III was referred to our hospital for treatment of severe aortic valve stenosis. Her other medical history included hypertension, chronic atrial fibrillation, arterial septal defect, and mild dementia. Transthoracic echocardiography showed severe aortic stenosis with concentric left ventricular hypertrophy, small arterial septal defect, and moderate pulmonary hypertension. The aortic valve area calculated by the continuity equation was $0.39 \mathrm{~cm}^{2}$ with a mean transvalvular gradient of $46 \mathrm{mmHg}$ (Figure 1A). The systolic tricuspid regurgitation (TR) pressure gradient was $71 \mathrm{mmHg}$, which indicated moderate pulmonary hypertension. Both the right and left atrium were enlarged. The left ventricular systolic function was normal. Coronary angiography showed a significant stenosis in the proximal right coronary artery. Right heart catheterization showed a mean right arterial pressure of 10 $\mathrm{mmHg}$, mean pulmonary artery pressure of $46 \mathrm{mmHg}$, and mean pulmonary wedge pressure of $20 \mathrm{mmHg}$. Cardiac output, determined by the Fick method, was 3.38 liter per 
minute. The calculated pulmonary to systemic blood flow ratio (Qp/Qs) was 1.14. The authors speculated that the patient's pulmonary hypertension was not explained by the combination of severe aortic stenosis and small arterial septal defect; involvement of primary pulmonary hypertension was suspected.

The patient was deemed high risk for surgical aortic valve replacement in view of the multiple comorbidities and frailty. TAVI was also considered high risk and its therapeutic value was considered uncertain due to the patient's pulmonary hypertension, which we considered not to be explained solely by the aortic stenosis. The authors chose to perform BAV to evaluate the therapeutic effect of opening the narrowed aortic valve. After obtaining the approval of our institutional human ethics committee and the written informed consent, BAV using the looped Inoue balloon technique was performed.

The device for the looped Inoue balloon technique was very similar as described in the preclinical animal study[6]. The technique employed a customized Inoue balloon catheter and two different stylets to make a loop in the left atrium. The size and shape of the heart are much different between healthy swine and diseased human. Thus, different sized stylets are required in the clinical use. A three-dimensional heart model based on the patient's computed tomographic (CT) scan data was made by laser stereolithography (crossEffect 
Inc., Kyoto, Japan). We conducted an in vitro simulation to decide the appropriate shape and size of the stylets (Figure 2). A large $1.0 \mathrm{~mm}$ stainless steel stylet with a loop of $22 \mathrm{~mm}$ radius and a $0.48 \mathrm{~mm}$ nitinol stylet with a loop of $5 \mathrm{~mm}$ radius were used in the present case (Figure 3).

Antegrade BAV using the looped Inoue balloon technique was performed under local anesthesia. The technique was essentially the same as previously described[6]. The diagrammatic representation of the looped Inoue balloon technique is depicted in Figure 4. After percutaneously placing a $6 \mathrm{~F}$ introducer sheath in the right femoral vein, a 0,035-inch guidewire was advanced to the left atrium thought the arterial septal defect. The guidewire was replaced to the 0.025 -inch coiled tip guidewire which is usually used in percutaneous transvenous mitral commissurotomy (PTMC). The customized Inoue balloon catheter was advanced to the left atrium over the 0.025 -inch guidewire placed in the left atrium, and then the two stylets were inserted. The balloon catheter was formed a loop shape by the two stylets. The customized Inoue balloon catheter was easily dropped into the left ventricle with a gentle rotation of the small stylet. The tip of the balloon catheter was placed just under the aortic valve. The large stylet was removed, and then a 0.035 -inch stiff guidewire was advanced to the ascending aorta thorough the narrowed aortic valve. The 0.035 -inch stiff 
guidewire was captured using a snare catheter and pulled into the descending aorta. The customized Inoue balloon catheter was advanced to the ascending aorta, and then balloon dilatation was conducted during rapid right ventricular pacing at 180 beats per minute. The balloon inflation was performed in a stepwise manner starting from $16 \mathrm{~mm}$ to $20 \mathrm{~mm}$ in diameter. Fluoroscopic images during the procedure were depicted in Figure 5. The procedure was successful without any adverse events. The procedure time was 100 minutes and the total amount of contrast medium was $30 \mathrm{ml}$. The patient's dyspnea improved after the procedure. Post-procedural transthoracic echocardiography demonstrated an improvement of aortic valve stenosis without worsening of aortic valve regurgitation (aortic valve area: $1.15 \mathrm{~cm}^{2}$, mean pressure gradient: $23 \mathrm{mmHg}$, Figure 1B). The systolic TR pressure gradient decreased to $54 \mathrm{mmHg}$. Both the patient's symptom and pulmonary hypertension were improved and has been maintained for 3 months after the BAV. The BAV proved that the opening of the narrowed aortic valve was very effective for the patient. TAV will be planned when the restenosis of aortic valve happens in the future.

\section{Discussion:}

The present paper describes the first clinical experience of the looped Inoue balloon 
technique in which two different sized stylets are used to make a loop in the left atrium. The size and shape of the stylets were determined in the pre-procedural simulation using a three dimensional heart model created from the patient's CT scan data. The procedure was successful without any complications. The present case indicates that the looped Inoue technique is feasible in humans.

BAV, introduced by Cribier et al in the 1980s[7], has played a niche role in the treatment of patients with severe aortic stenosis. The application of BAV had been limited in patients who were not a good candidate for surgical aortic valve replacement because of the high complication and restenosis rates. However, BAV is performed more commonly at present as a bridge therapy to $\operatorname{TAVI}[8,9,2,10]$. BAV also plays a vital role in selecting a patient who is suitable for TAVI. We conducted BAV in the present case to clarify the treatment effect of opening the narrowed aortic valve.

BAV can be performed by either the retrograde transarterial approach or the antegrade transvenous approach to the aortic valve. The antegrade approach, which was first performed by Inoue et al.[11], has several advantages over the retrograde approach[3, 4, 5]. The antegrade approach reduces vascular complications since it uses venous access site. Venous access also enables the use of larger balloons, resulting in greater increase 
post-BAV valve area [3]. However, the retrograde approach is more frequently employed in most institutions because the antegrade approach is more complicated, technically demanding, and expensive. The antegrade approach requires transseptal puncture and a guidewire placement to make a loop in the left ventricle. The step in making a loop in the left ventricle with avoiding damage to the mitral apparatus, mechanical stress to the ventricular wall, and arrhythmia is sometimes difficult. The looped Inoue balloon technique simplifies the antegrade BAV by making a loop in the left atrium. The looped Inoue balloon can be easily advanced to the left ventricle by handling the stylets. Making a loop in the left atrium is reasonable because the inner surface of the left atrium is much smoother than that of the left ventricle. Furthermore, there are no structural obstacles including chordae tendineae, papillary muscle and mitral valve leaflets.

In conclusion, the present case report demonstrates the feasibility of the looped Inoue balloon technique in humans. Efficacy and benefits of this technique should be assessed in a large number of patients.

\section{Acknowledgement:}


The authors wish to thank Mr. Mitsuru Sato and Mr. Yoshimasa Nagata for the technical assistance in developing the catheter system. We would also like to express our gratitude to Mr. Kinnya Akimoto, Mr. Katsunori Hatanaka and Mr. Masatoshi Takeda for making the heart model. Special thanks also go to Ms. Rika Sugiura, Ms. Kotoko Omichi and Ms. Yoko Shimizu for their secretarial assistance.

\section{Conflict of Interest:}

Dr. Kanji Inoue holds all patents of the balloon catheter described in the present report. The other authors have no conflict of interest to declare.

\section{References:}

1. Ben-Dor I, Maluenda G, Dvir D, Barbash IM, Okubagzi P, Torguson R, Lindsay J, Satler LF, Pichard AD, Waksman R (2013) Balloon aortic valvuloplasty for severe aortic stenosis as a bridge to transcatheter/surgical aortic valve replacement.

Catheter Cardiovasc Interv 82:632-637 
2. Hara H, Pedersen WR, Ladich E, Mooney M, Virmani R, Nakamura M, Feldman T, Schwartz RS (2007) Percutaneous balloon aortic valvuloplasty revisited: time for a renaissance? Circulation 115:e334-338

3. Sakata $Y$, Syed Z, Salinger MH, Feldman T (2005) Percutaneous balloon aortic valvuloplasty: antegrade transseptal vs. conventional retrograde transarterial approach. Catheter Cardiovasc Interv 64:314-321

4. Eisenhauer a C, Hadjipetrou P, Piemonte TC (2000) Balloon aortic valvuloplasty revisited: the role of the inoue balloon and transseptal antegrade approach. Catheter Cardiovasc Interv 50:484-491

5. Cubeddu RJ, Jneid H, Don CW, Witzke CF, Cruz-Gonzalez I, Gupta R, Rengifo-Moreno P, Maree AO, Inglessis I, Palacios IF (2009) Retrograde versus antegrade percutaneous aortic balloon valvuloplasty: immediate, short- and long-term outcome at 2 years. Catheter Cardiovasc Interv 74:225-231

6. Bao B, Saito N, Watanabe S, Tokushige A, Yamamoto E, Kawase Y, Kimura T, Inoue K (2013) A novel device for antegrade percutaneous balloon aortic valvuloplasty: 
feasibility of the looped Inoue balloon technique in swine model. Catheter Cardiovasc Interv 82:E564-568

7. Cribier A, Savin T, Saoudi N, Rocha P, Berland J, Letac B (1986) Percutaneous transluminal valvuloplasty of acquired aortic stenosis in elderly patients: an alternative to valve replacement? Lancet 1:63-67

8. Furuta A, Shimizu H, Hachiya T, Ohno Y, Kawajiri H, Kawamura A, Kokaji K, Yozu R, Fukuda K (2013) Time to revisit role of transcatheter balloon aortic valvuloplasty: a bridge-therapy to subsequent treatment case report. Heart Vessels 28:397-400

9. Maekawa Y, Kawamura A, Furuta A, Yuasa S, Fukuda K (2012) A case of severe aortic stenosis with severe coronary artery disease that was successfully treated by balloon aortic valvuloplasty and percutaneous coronary intervention. Heart Vessels 27:528-531

10. Sack S, Kahlert P, Khandanpour S, Naber C, Philipp S, Möhlenkamp S, Sievers B, Kälsch H, Erbel R (2008) Revival of an old method with new techniques: balloon aortic valvuloplasty of the calcified aortic stenosis in the elderly. Clin Res Cardiol 97:288-297 
11. Inoue K, Nobuyoshi M, Mitudo K, Saito S, Nakamura N, Tamai H, Watanabe S, Suzuki T, Koga N (1988) Percutaneous Transvenous Aortic Valvuloplasty by a Self-Positionig Balloon (Inoue-Balloon). J Am Coll Cardiol 11:221A(1A-256A)

\section{Figures Captions}

Figure 1

Pre- and post-procedural transthoracic echocardiography. The peak transvalvular velocity decreased from $4.38 \mathrm{~m} / \mathrm{s}(\mathrm{A})$ to $3.3 \mathrm{~m} / \mathrm{s}(B)$. The aortic valve area increased from $0.39 \mathrm{~cm}^{2}$ to $1.15 \mathrm{~cm}^{2}$.

\section{Figure 2.}

Three dimensional hear model and in vitro simulator system. A: Anterior view of a threedimensional CT reconstruction image of the heart. B: The three-dimensional heart model based made by laser stereolithography. C, D, E: In vitro simulator system. The heart model is connected to a pump creating a pulsatile flow. The simulation was conducted under 
fluoroscopy.

Figure 3.

The catheter system for the looped Inoue balloon technique. A: The two different sized stylet employed in the catheter system. The large stylet is made of $1.0 \mathrm{~mm}$ stainless with a $22 \mathrm{~mm}$ radius. The small stylet is made of $0.48 \mathrm{~mm}$ nitinol with a $5 \mathrm{~mm}$ radius. $B$. The proximal part of the balloon catheter. The large stylet is inserted into the central lumen (arrow). The small stylet is inserted into the extra port (arrow head). C. The distal end of the balloon catheter is the same to the original Inoue balloon catheter.

\section{Figure 4.}

The diagrammatic representation of the looped Inoue balloon technique. 1: The balloon catheter is advanced into the left atrium after the transseptal puncture. 2: The two stylets is inserted in the catheter to make a loop in the left atrium. The balloon catheter is dropped into the left ventricle by pushing the balloon catheter with a counter clockwise rotation of the small stylets. 3: A stiff guidewire is advanced to the descending aorta via the narrowed aortic 
valve. 4: The balloon catheter is advanced to the ascending aorta.

Figure 5.

Fluoroscopic images during the procedure. 1: The balloon catheter was advanced to the left atrium after the transseptal puncture. 2: The balloon catheter is formed a loop by inserting the two stylets. 3: After dropping the balloon catheter by handling the stylets, a 0.035-inch stiff guidewire was advanced to the ascending aorta. The guidewire was caught by a snare catheter inserting from the left femoral artery. 4: The balloon catheter was advanced to cross the aortic valve. Balloon was inflated at the aortic valve. 


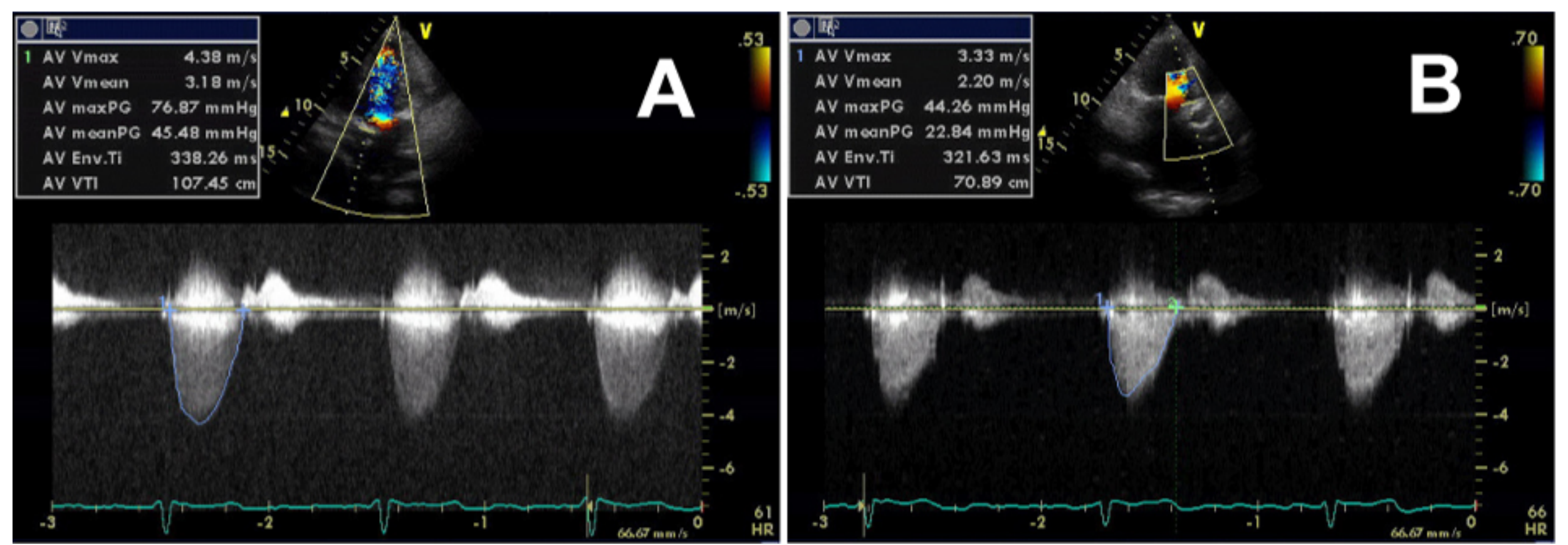

Figure 1 

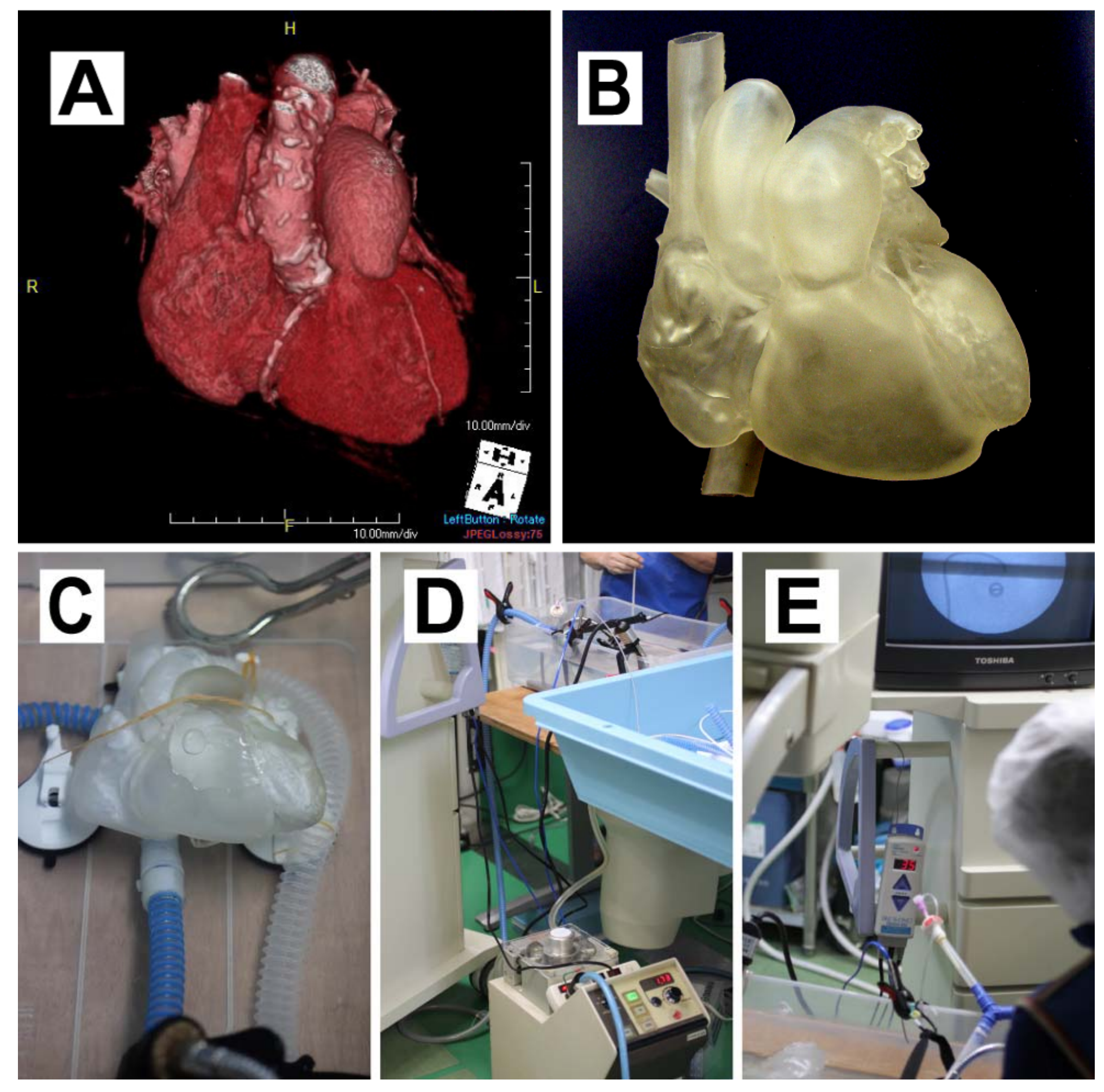

Figure 2 


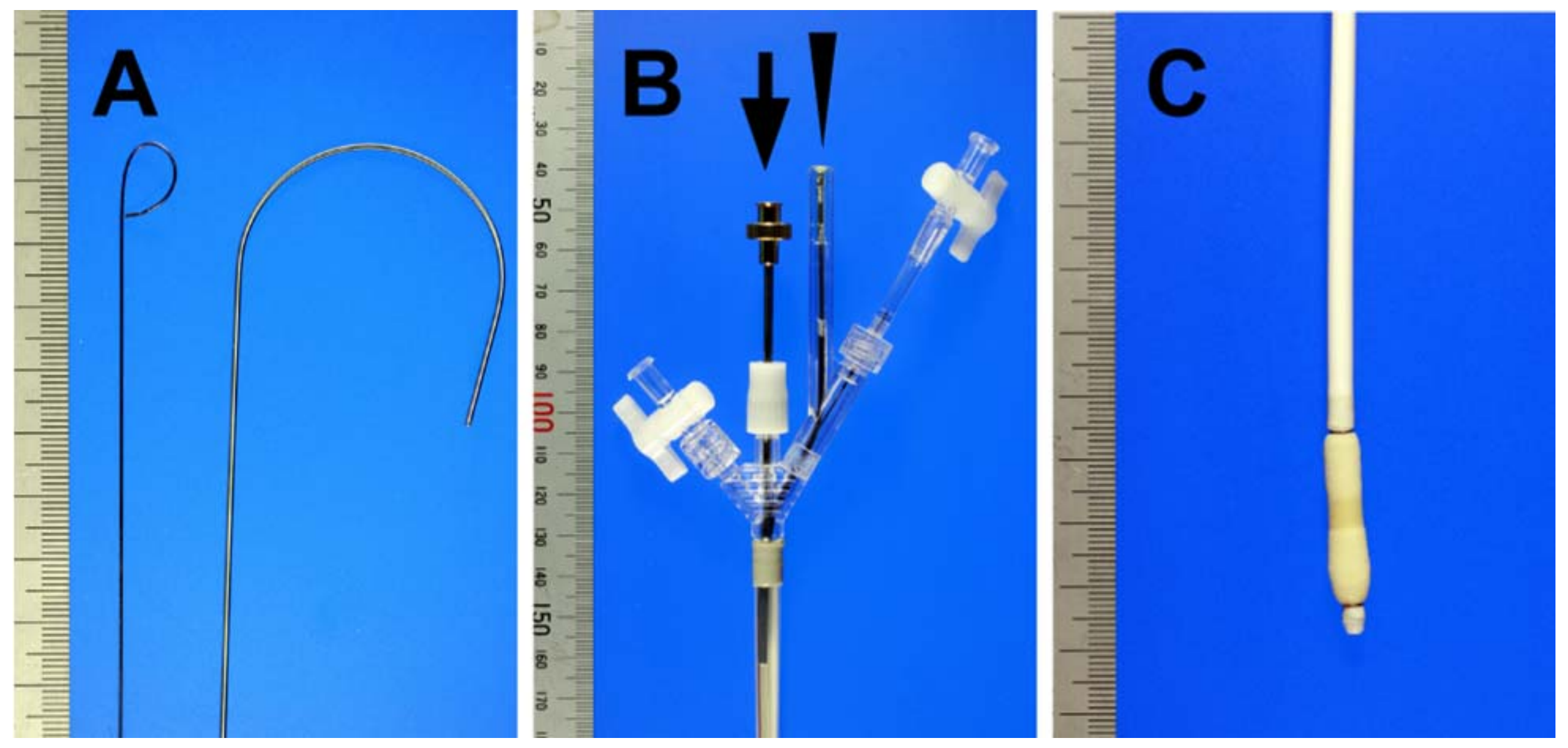

Figure 3 


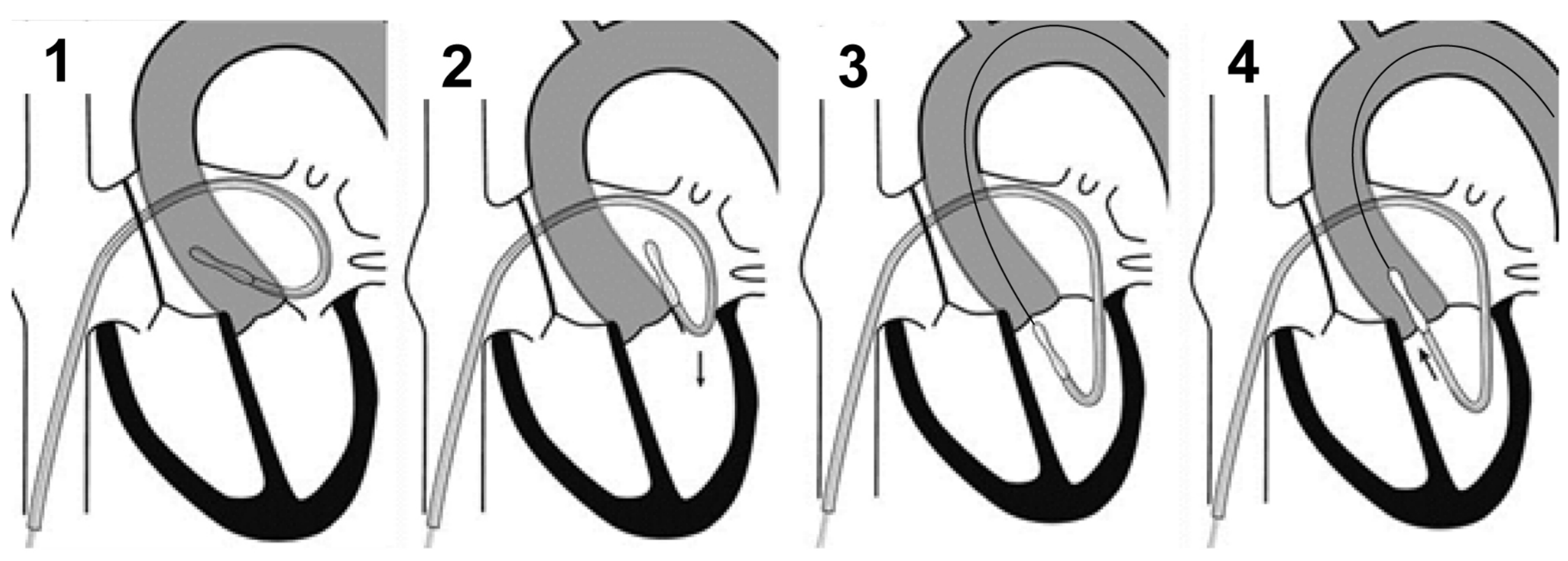

Figure 4 


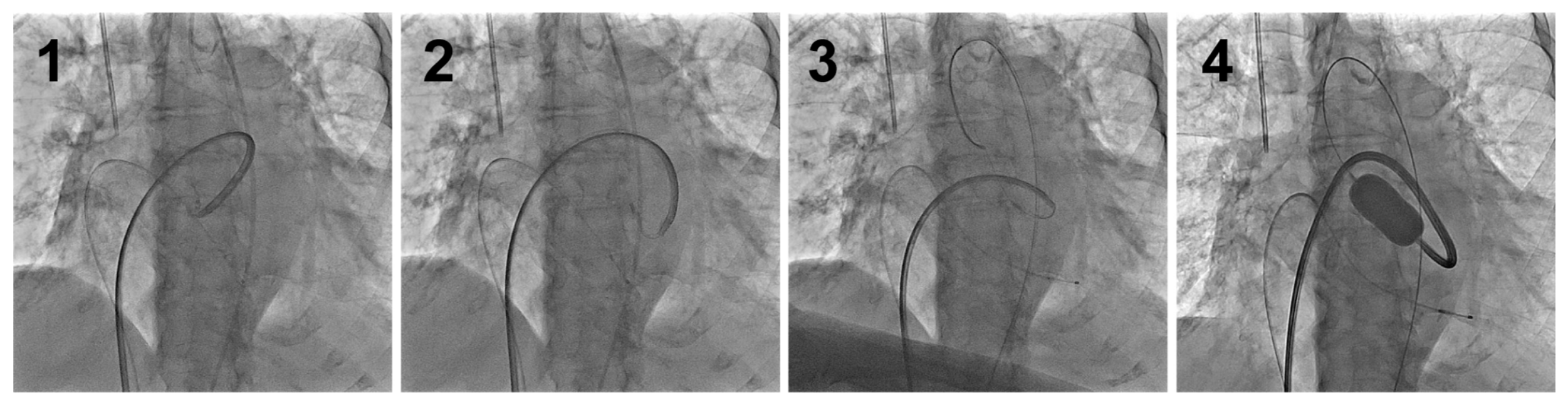

Figure 5 